\title{
Chiesa, Religione e Monarchia negli Stati italiani preunitari. \\ Intransigenti e reazionari fra Trono e \\ Altare (1820-1830)
}

\section{Church, Religion and Monarchy in the Pre-unitary Italian States. Intransigents and Reactionaries between Throne and Altar (1820-1830)}

\author{
Nicola Del Corno \\ Università degli Studi di Milano \\ nicola.delcorno@unimi.it
}

Recibido: 7-V-2015

Aceptado: 3-IX-2015

\section{Resumen}

En los primeros años de la Restauración, en Italia se verificó un juego sutil de alianzas entre gobiernos, corrientes ultra-católicas y fuerzas reaccionarias, con tendencia a la represión de las posiciones progresistas y al refuerzo del poder político. Los gobiernos concedieron una cierta libertad al catolicismo inflexible, para que pudiera aumentar un renovado tipo de proselitismo con la intención de acercar a una concepción más dogmática de la religión a quien habían estado desviado de sugestiones subversivas. Por su parte, la reacción laica, por cuenta sobre todo de los soberanos, se empeñó en una ferviente batalla de propaganda contra liberales y moderados. Por lo tanto, la política y la religión terminaron por unirse en un vínculo inseparable que no permite delimitar los respectivos campos de acción, de modo que una instrumentalizaba a la otra, para conseguir formar un frente común ultraconservador.

Palabras clave: Monarquía, Religión, Iglesia, Italia, siglo XIX.

\section{Abstract}

During the first years of the European Restoration, a subtle game of alliances between governments, ultra-catholic currents and reactionary forces took place in Italy. This interaction aimed to repress the progressive demands while strengthening the political 
power originated from the Congress of Vienna. Thus the governments accommodated intransigent Catholicism with a certain freedom in order to encourage a revived proselytism. This had the main purpose to draw back to a dogmatic form of religion those who had been influenced by subversive trends. On the contrary, the secular response, proposed mainly by the sovereigns, waged a fierce propagandistic battle against the liberals and the moderates. As a consequence, politics and religion became inextricably linked and their respective fields of action encroached upon one another. Hence, politics and religion took advantage of each other in their common ultraconservative intent.

Keywords: Monarchy, Religion, Church, Italy, $19^{\text {th }}$ Century.

Nel 1821, dalle colonne della Enciclopedia ecclesiastica il padre teatino Gioacchino Ventura poneva l'esperienza religiosa al di sopra di qualsiasi altra manifestazione umana, compresa quella politico-sociale. Il potere della Chiesa infatti, originato dall'assolutezza della verità rivelata, sussisteva indipendentemente dal sostegno dei governi; questi, al contrario, svuotati del fondamento morale della fede, non potevano affrancarsi dall'incerta contingenza della propria mondanità: "LAltare senza l'appoggio del Trono può sostenersi, poiché ergesi sopra quell'immobil sasso, contro del quale gli sforzi riuniti di tutto l'abisso, non prevarranno giammai; ma il trono, cui la religione cessa di prestare il suo appoggio; l'autorità a cui essa non imprime la sua divina sanzione; l'ubbidienza che essa non santifica con la santità dei motivi... che diverranno essi mai?"1.

Nonostante da parte dell'intransigentismo cattolico si pronunciasse ${ }^{2}$, come si è visto, la superiorità dell'aspetto religioso su quello meramente politico, e di conseguenza si richiedesse una maggiora autonomia e indipendenza delle istituzioni religiose negli affari della società così come dello Stato, si venne a creare nei primi anni della Restaurazione in Italia un sottile, ma almeno da principio proficuo, gioco di alleanze fra governi, correnti ultracattoliche e forze reaziona-

1. Ventura, Gioacchino, "Religione pubblica. Vicende della religione", Enciclopedia ecclesiastica e morale, vol. I (1821), pp. 26-27. Su Ventura esiste una vasta bibliografia; ci si limita pertanto a segnalare, ANDREU, Francesco, "P. Gioacchino Ventura. Saggio biografico", Regnum Dei, vol. XVII (1961), pp. 1-161; TesinI, Mario, Gioacchino Ventura. La Chiesa nell'età delle rivoluzioni, Roma, Edizioni Studium, 1988; CARONITI, Dario, Potere pubblico, tradizione e federalismo nel pensiero politico di Gioacchino Ventura, Soveria Mannelli, Rubbettino, 2014; e gli atti del convegno, Gioacchino Ventura e il pensiero politico d'ispirazione cristiana dell'Ottocento, a cura di E. Guccione, Firenze, L.S. Olschki, 1991, 2. voll.

2. Per un quadro più ampio sul cattolicesimo italiano in età contemporanea, e non relativo solo all'intransigentismo, si rimanda ai due classici studi, l'uno di ispirazione marxista, CANDEloro, Giorgio, Il movimento cattolico in Italia, Roma, Rinascita, 1953; l'altro di ispirazione cattolica, De RosA, Gabriele, Storia del movimento cattolico in Italia, Bari, Laterza, 1966, 2 voll. 
rie, tendente alla repressione delle istanze innovative e al rafforzamento del potere politico scaturito dal Congresso di Vienna. I governi concessero così una certa libertà al cattolicesimo intransigente perché incrementasse un nuovo tipo di proselitismo volto a riavvicinare ad una concezione più dogmatica della religione chi era stato sviato da suggestioni di tipo razionalistico ed individualistico. La Chiesa mirava, inoltre, a non essere ostacolata in quella operazione di recupero presso la società civile di quelle posizioni di prestigio e di potere che erano state scosse dagli eventi rivoluzionari. Dal canto suo, la reazione laica, per conto soprattutto dei sovrani legittimi, e godendo di un sostegno politico più o meno esplicito da parte dei rispettivi governi, s'impegnava in una fervente battaglia ideologica e propagandistica per un verso contro democratici, liberali e moderati, per l'altro contro la preponderante egemonia austriaca negli affari italiani; un'opposizione che tuttavia risulterà sempre meno marcata quando ci si accorse dell'assoluta necessità dell'intervento armato asburgico per contenere i moti rivoluzionari ${ }^{3}$.

Se nel lessico politico per reazione s'intende ogni atteggiamento che, opponendosi ad un processo evolutivo, tenta di arrestarlo proponendo un ritorno al passato, anche il pensiero controrivoluzionario italiano -rappresentato in buona parte da cattolici intransigenti- non si propose certamente come una corrente dottrinaria autonoma e originale, ma si sviluppò come una decisa risposta a manifestazioni pragmatiche e teoriche ad essa opposte, prima fra tutte ovviamente la Rivoluzione francese, e poi i diversi moti insurrezionali che caratterizzarono il processo risorgimentale nel nostro paese. Va sottolineato però come i propugnatori di questa parte politica, anziché definirsi reazionari, ultraconservatori o controrivoluzionari, preferirono sempre denominarsi come legittimisti, rimarcando la fedeltà ad un ordine tradizionale legittimato in prima istanza da Dio ('Altare risulta quindi il supremo disciplinatore della società umana), e poi dalla storia e dalla consuetudine, e che prevedeva l'inamovibilità delle dinastie regnanti sul suolo italiano secondo un preciso disegno divino ${ }^{4}$.

È facilmente riscontrabile, nel considerare cronologicamente le alterne fortune di questo movimento in Italia fra la Rivoluzione francese e il compimento

3. ARA, Angelo, Le correnti conservatrici in Italia, in Istituzioni e ideologie in Italia e in Germania tra le rivoluzioni, a cura di U. Corsini e R. Lill, Bologna, il Mulino, 1987, pp. 95-96; DEL CORno, Nicola, Gli "Scritti sani". Dottrina e propaganda della reazione italiana dalla Restaurazione all'Unità, Milano, Franco Angeli, 1992, p. 13.

4. Per le vicende del legittimismo italiano si rimanda a LEONI, Francesco, Storia della controrivoluzione in Italia (1799-1859), Napoli, Guida, 1975; per una sintesi del pensiero politico espresso a Del Corno, Nicola, Reazione, in Atlante culturale del Risorgimento. Lessico del linguaggio politico dal Settecento all'Unità, a cura di A.M. Banti, A. Chiavistelli, L. Mannori, M. Meriggi, Bari-Roma, Laterza, 2011, pp. 163-175. 
del processo risorgimentale, come la polemica reazionaria abbia trovato una propria eco presso l'opinione pubblica soprattutto in conseguenza, e come diretta risposta, al ripetersi di tentativi insurrezionali compiuti da liberali e democratici. Il succedersi di rivoluzioni, governi liberali, restaurazioni assolutiste, con lo sbocco finale nella creazione dello Stato italiano, fece sì che la difesa del passato venisse assunta con un seguito non irrilevante da chi si era sempre opposto ad ogni novità, e riteneva di non essere mai stato ascoltato in maniera soddisfacente da quelle autorità che avrebbero dovuto tenere più in considerazione gli ammonimenti a non lasciare alcuno spazio ai prodotti della modernità politica e culturale.

I moti del 1820-21 nel Regno delle Due Sicilie e in quello sabaudo scatenarono, oltreché una decisa repressione armata da parte dei governi assoluti, anche una risoluta controffensiva ideologica, nella quale giocarono un ruolo di primo piano principalmente tre riviste -la napoletana Enciclopedia ecclesiastica e morale, le modenesi Memorie di Religione, di Morale e di Letteratura e la torinese Amico d'Italia- che pur se principalmente d'ispirazione religiosa non lesinavano nelle loro pagine palesi riferimenti alla situazione politico-sociale coeva secondo una prospettiva legittimista e antiliberale. Questa pubblicistica mirava a combattere principalmente la diffusione delle dottrine razionalistiche ed antropocentriche, e in nome di un ferreo dogmatismo confutava ogni errore di natura teologica, considerandolo prodromo di ben più pericolosi inganni politici. Pur basandosi su presupposti dell'ortodossia cattolica e non propriamente su istanze politiche, inevitabilmente il dibattito interno a questa stampa tese a spostarsi di frequente anche su un terreno socialmente più esteso, coinvolgendo vari aspetti del vivere collettivo. Di conseguenza politica e religione finirono per risultare legate da un vincolo inscindibile tale da non permettere di delimitare in maniera netta i rispettivi campi d'azione, cosicché l'una finiva per strumentalizzare l'altra e viceversa, per arrivare ad una comune finalità di indirizzo ultraconservatore ${ }^{5}$. La stretta interdipendenza fra religione e politica fu sicuramente un cavallo di battaglia nella polemica reazionaria di allora: "nell'ordine religioso è perfettamente nullo tutto ciò che non è cattolico, come è nullo tutto ciò che non è monarchico nell'ordine politico" scriveva infatti nel 1825 il già citato Ventura ${ }^{6}$, suscitando le critiche degli avversari che avevano gioco facile nell'accusare i controrivoluzionari di con-

5. Galante Garrone, Alessandro, I giornali della Restaurazione 1815-1847, in La stampa italiana del Risorgimento, Bari, Laterza, 1979, p. 101.

6. Ventura, Gioacchino, "Della disposizione attuale degli spiriti in Europa rispetto alla religione e della necessità di propagandare i buoni principi per mezzo della stampa", Giornale Ecclesiastico, vol. III (1825), p. 39; tale articolo fu pubblicato nello stesso anno anche sulle modenesi Memorie di Religione, di Morale e di Letteratura, vol. VII (1825), pp. 385-421. 
siderare la religione quale instrumentum regni, degradandola così a organo di coercizione e di controllo ${ }^{7}$.

Nei contenuti di questi giornali notevole fu l'influenza transalpina ${ }^{8}$; la Francia teocratica di Joseph de Maistre e quella antiindividualista di Louis de Bonald da un punto di vista ideologico, quella delle missioni da un punto di vista pratico risultarono un riferimento costante. Ma fu soprattutto Félicité de Lamennais a fornire un intero arsenale di idee, polemiche, suggestioni, tale da tentare anche nel nostro paese una sorta di controrivoluzione intellettuale, di cui appunto la teoria del sensus communis dell'abate bretone risultava l'asse portante per far partire la riconquista cattolica dell'intera società italiana ${ }^{9}$.

La preponderante influenza transalpina sugli ultracattolici italiani è stata messa in luce dalla storiografia che si è occupata dello studio delle riviste sopraccitate, e ci si riferisce soprattutto a Sandro Fontana, Anna Foa e Anna Maria Battista ${ }^{10}$; anche se, secondo quest'ultima, in realtà "il pensiero di Maistre ${ }^{11}$,

7. VerucCI, Guido, Per una storia del cattolicesimo intransigente in Italia dal 1815 al 1848, in I cattolici e il liberalismo. Dalle "Amicizie cristiane" al modernismo, Padova, Liviana, 1968, p. 16.

8. Sul ben più vivace movimento ultracattolico francese esiste una vasta bibliografia; per limitarsi a qualche titolo si veda, oltre al classico RÉMOND René, La droite en France de 1815 à nos jours, Paris, Aubier, 1954, soprattutto REARDON Bernard, Liberalism and Tradition. Aspects of Catholic Thought in Nineteenth Century France, Cambridge, Cambridge University Press, 1975; SERRA Teresa, L'utopia controrivoluzionaria. Aspetti del cattolicesimo "antirivoluzionario" in Francia (1796-1830), Napoli, Guida, 1977; GENGEMBRE Gerard, La Contre-revolution ou l'histoire désespérante, Paris, Imago, 1989; DenIs Michel, 18151848. Que faire de la Révolution française?, in Histoire des droites en France, sous la direction de J.-F. Sirinelli, Paris, Gallimard, 1992, vol. I Politique, pp. 13-88.

9. Come ebbe modo di notare GAMBARO, Angiolo "il periodo di più esteso successo del Lamennais in Italia fu quello teocratico puro, quando l'immobilismo imposto dalla Santa Alleanza, le tendenze sistematicamente reazionarie della Restaurazione e certe istituzioni contemporanee di propaganda religiosa parevano fatti apposta per favorirlo", "La fortuna di Lamennais in Italia", Studi francesi, a. II (1958), p. 207. Sull'influenza di Lamennais nel pensiero politico italiano si vedano soprattutto dello stesso GAMBARO, oltre all'articolo sopra ricordato, Sulle orme di Lamennais in Italia. Il lamennesismo a Torino, Torino, Deputazione subalpina di storia patria, 1958; VerucCI, Guido Félicité Lamennais. Dal cattolicesimo autoritario al radicalesimo democratico, Napoli, Istituto per gli studi storici, 1963; e SANCIPRIANO, Mario, Lamennais in Italia. Autorità e libertà nel pensiero filosofico e religioso del Risorgimento, Milano, Marzorati, 1973.

10. FONTANA, Sandro La controrivoluzione cattolica in Italia (1820-1830), Brescia, Morcelliana, 1968; FoA, Anna, Gli intransigenti, la Riforma e la Rivoluzione francese. Un dibattito nella pubblicistica italiana dell'età della Restaurazione, L'Aquila, Japadre, 1975; BATTISTA, Anna Maria, Aspetti del tradizionalismo italiano nell'età della Restaurazione, in La Restaurazione in Italia. Strutture e ideologie. Atti del XLVII Congresso di storia del Risorgimento italiano, Roma, Istituto per la storia del Risorgimento, 1975 pp. 223-249.

11. Come già la Battista, anche GALLI, Carlo (I controrivoluzionari, Bologna, il Mulino, 1981) e SERRA, Teresa (L'utopia controrivoluzionaria...) hanno considerato il sabaudo Joseph de Maistre quale esponente della cultura politica francese. 
di Bonald, di Lamennais fu scarsamente recepito in Italia, ovvero venne letto in una ottusa chiave reazionaria", e pertanto rimase "congelato negli scritti di alcuni pensatori" -soprattutto Gioacchino Ventura- "le cui tesi trovarono riscontri scarsi ed episodici", non in grado quindi di influenzare la cultura italiana di parte cattolica ${ }^{12}$. Secondo la Foa fattori decisivi per decretare "la maggiore maturità" del pensiero reazionario francese su quello italiano risultavano non solo una ben più consistente tradizione culturale, ma soprattutto il diverso contesto pragmatico, dato che i francesi avevano vissuto concretamente una complessa situazione storica, politica e religiosa in movimento, che aveva visto il succedersi di Illuminismo, Rivoluzione francese, periodo napoleonico, mentre gli italiani spesso si limitavano ad "una astratta elaborazione teorica", di conseguenza "non originale", e ciò determinò secondo l'autrice "il sostanziale fallimento" della polemica intransigente italiana, incapace di andare oltre ad una stantia battaglia contro i lasciti del XVIII secolo ${ }^{13}$. Da parte sua Fontana metteva in luce come questa influenza francese fosse "netta e poderosa", tale da far risultare pochi "i contributi autonomi e originali" prodotti dagli intransigenti italiani a tal punto che risulta "piuttosto meccanico lo sforzo di mediazione tra schemi importati e tradizioni e realtà locali"14.

Come sottolineato da Guido Verucci, agli inizi degli anni '20 l'intransigentismo italiano guardava alla Francia come a una nazione, che se pure era stata culla dell'empia filosofia illuminista scatenante il fenomeno rivoluzionario, era in seguito diventata motore della riscossa restauratrice da un punto di vista religioso, e per questo motivo veniva ora contrapposta in positivo dai reazionari italiani all'Inghilterra, animatrice sì dello schieramento antinapoleonico e quindi, secondo la loro ottica, controrivoluzionario, ma pur sempre nazione protestante, costituzionale e liberale ${ }^{15}$. A dimostrazione di questa nuova francofilia caratterizzante il microcosmo legittimista del nostro paese, Verucci ricordava un passaggio tratto dalla già citata Enciclopedia Ecclesiastica in cui si rimarcava come fosse "consolante il vedere che la Francia, la prima a scuotere il giogo soave della Religione, è ancora il primo a ripigliarlo"16.

Dai pensatori transalpini, i reazionari italiani attingevano soprattutto il mito della Restaurazione incompiuta; questa infatti avrebbe dovuto risultare integrale comportando quale condizione imprescindibile il ritorno del cristiane-

12. Battista, Anna Maria, Aspetti del tradizionalismo..., p. 249.

13. FOA, Anna, Gli intransigenti..., p. 11.

14. Fontana, Sandro La controrivoluzione cattolica..., p. 7, e p. 13.

15. Verucci, Guido, Per una storia del cattolicesimo intransigente..., p. 22.

16. Ventura, Gioacchino, "Osservazione sopra i dibattimenti precedenti", Enciclopedia ecclesiastica e morale, vol. I (1821), p. 213. 
simo al primigenio stato di unità, così come lo era stato prima della scissione religiosa luterana. Non ci si poteva accontentare di una Restaurazione limitata al contingente compito di risistemazione politica in senso legittimista dell'Europa dopo le temperie rivoluzionarie e napoleoniche; diveniva fondamentale, per prevenire nuove insurrezioni d'ogni ordine, ritornare a quella tradizionale unità di fede europea, unica condizione certa di pace e prosperità per il continente. Se la Rivoluzione francese era stata la logica conseguenza di una lunga età di crisi iniziata con la Riforma, funestata dalle guerre di religione, e di conseguenza caratterizzata dalle incertezze del comando politico in molte nazioni, la Restaurazione doveva assumere il significato primario di inizio di una nuova epoca d'ordine caratterizzante il futuro europeo ${ }^{17}$.

In quest'ottica di polemica antiprotestante, notevole spazio sulla stampa cattolica italiana venne dato al tema delle conversioni religiose che stavano colpendo il mondo riformato; si riportavano infatti numerosi casi di protestanti illustri -primo fra tutti Karl-Ludwig von Haller- che avevano deciso di ritornare nell'accogliente casa della Chiesa romana. Anche in questo caso si trattava di una ripresa del modello francese, dove in alcune riviste vi erano delle vere e proprie rubriche dedicate a queste conversioni -i Recuil de conversione remarquables ${ }^{18}$ - il cui scopo era quello di dimostrare la vastità, ma soprattutto la continuità di un fenomeno teso a prosciugare il protestantesimo dei suoi esponenti intellettuali migliori. Sulla stampa italiana, si rimarcava come queste conversioni fossero volontarie, dettate dalla scoperta della linea di stretta continuità che vi era stata fra Riforma e Rivoluzione in una comune critica sovversiva contro le autorità costituite. Dalle colonne delle Memorie di religione tale stretto nesso fu messo in luce dallo stesso Ventura, allorché faceva notare come la Riforma fosse nata proprio dalla volontà di rivoltarsi contro ogni principio d'ordine creando quell'anarchia nel mondo morale propedeutica per conseguenti sollevazioni in ambito politico: la democrazia altro non era infatti che un "vero protestantesimo nello Stato"; e d'altro canto il protestantesimo appariva come "una vera democrazia nella ragione". Così ribadiva il padre teatino a supporto della sua accusa al protestantesimo di aver fomentato quello spirito d'insubordinazione pratica e teorica che avrebbe poi portato inevitabilmente all'empietà dei fatti parigini del 1789:

"La Rivoluzione religiosa e politica, che da tre secoli desola la terra, non è cominciata in Europa che da una guerra generale mossa contro tutti i principi dell'ordine, il quale è il riposo delle intelligenze ed il gran costitutivo della

17. FOA, Anna, Gli intransigenti..., p. 151.

18. Ibid., p. 63. 
società. [...] La gran legge dell'ubbidienza degli spiriti al medesimo spirituale potere, cioè la fede, questa legge che è l'unico legame il quale unisce e mette in armonia fra loro le intelligenze nel mondo morale, come la legge dell'attrazione mantiene ne' giusti loro rapporti i corpi del mondo fisico, questa legge, dico, dichiarata una usurpazione ingiusta de' naturali diritti dell'uomo, fu solennemente abolita, e con essa fu legalmente sbandito l'ordine della società. [...] Allora fu legalizzata la democrazia, che è vero protestantesimo nello Stato, ed il protestantesimo, che è un vera democrazia nella ragione"19.

Risultava pertanto principalmente un giudizio politico quello espresso dai reazionari italiani su tale fenomeno; i convertiti non erano presentati come degli eretici fulminati di colpo dalla grazia divina, ma come buoni e giudiziosi sudditi, che devoti alle sorti delle loro legittime monarchie, avevano compreso come queste avessero bisogno del supporto del cattolicesimo, quale collaudato baluardo contro ogni tipo di deriva sovversiva, mentre il protestantesimo si rivolgeva, e soprattutto si addiceva, a chi voleva rivoluzionare le fondamenta del vivere collettivo.

Pertanto Giuseppe Baraldi s'impegnò sulle Memorie da lui dirette in una strategia mediatica tesa a dimostrare come il Protestantesimo iniziasse ad essere sconfessato, dopo i lutti e le distruzioni causati dal suo diretto prodotto rivoluzionario, dai propri fedeli in buona parte dell'Europa. Cominciò a trovare spazio, soprattutto nelle pagine della rivista modenese, una lunga serie testimonianze, quasi sempre tratte direttamente dalla stampa estera, sulle conversioni al cattolicesimo di personaggi più o meno illustri che stavano mettendo in crisi le fondamenta dello schieramento riformatore.

Fra i più noti, ai quali le Memorie dedicarono spazio e rilevanza, vanno ricordati, oltre al già citato Karl-Ludwig von Haller, Pierre de Joux e Friedrich Leopold Stolberg. Particolarmente significativa risultava la testimonianza di quest'ultimo, volta a dare del protestantesimo l'immagine di una religione implacabilmente in declino, perché già tarata di suo da una sostanziale mancanza di fede destinata a portare all'ateismo, e quindi al suo autoannullamento; scriveva infatti lo Stolberg, e riportava le sue parole a mo' di monito il giornale modenese:

"Nato protestante, io ero veramente tale, e con rammarico vedevo il protestantesimo dar crollo. Esso ruina senz'urto, seguendo la sua propria tendenza; corrompevasi per una sorta di corruttela tutta sua propria. Il suo nome stesso di protestantesimo, nome parlante, perché è negativo, annunzia uno spirito inquieto, torbido, tendente a distruggere non a stabilire. Ben presto volse contro

19. Ventura, Gioacchino, "Della disposizione attuale"..., pp. 23-24. 
sé le proprie armi, spogliossi delle auguste verità, che avea pur rispettate, le cangiò in altrettanti dubbi, ed eccolo presso a finire, dando un gran passo verso l'ateismo"20.

Per gli scopi propagandistici delle Memorie però non meno importante era stata la confessione dell'uomo politico francese de Joux il quale aveva ammesso in un saggio, ripreso in traduzione, che "la Rivoluzione religiosa del XVI secolo è la causa principale della rivoluzione politica scoppiata nel 1789", ossia -concludeva l'autore- del "sistema più vasto di distruzione del social ordine, che siasi mai offerto al mondo atterrito, e dal quale un concorso inudito di circostanze su di cui è impressa la mano di Dio, ha solo potuto liberarci"21; motivo essenzialmente politico per il quale il de Joux aveva deciso di convertirsi al cattolicesimo.

Inoltre particolare rilevanza fu data dalla stampa cattolica italiana, tramite una fitta serie di benevole recensioni, all'opera del polemista radicale inglese William Cobbet, Storia della Riforma protestante in Inghilterra e in Irlanda, uscita nel 1824 e immediatamente tradotta in italiano per volere dello stesso pontefice Leone $\mathrm{XII}^{22}$, nel corso della quale si denunciava come la Riforma fosse risultata la principale causa del generale impoverimento della società, soprattutto in Irlanda, poiché era andata a colpire quelle strutture monastiche di assistenza ai ceti subalterni, proprie della tradizione cattolica. Secondo Cobbet, alla base dello sviluppo capitalistico inglese, e dei conseguenti terribili problemi di pauperismo prima ignoti, vi era quella rivoluzione industriale che aveva avuto dalla sua una sorta di supporto etico da parte della Riforma, colpevole di voler stravolgere i collaudati equilibri della società, anche tramite una metodologia economica tendente alla frantumazione della solidarietà intercetuale da sempre auspicata dalla parola di Dio, per cui occorreva aiutare il proprio prossimo e non porsi nei suoi confronti in un'ottica di spietata concorrenza.

Anche alla luce di queste considerazioni si può comprendere come nella stampa in questione non vi fosse alcun timore di un possibile contagio riformato in Italia; se si denunciavano i tentativi di penetrazione nel tessuto sociale italiano compiuto dalla propaganda delle Società bibliche tramite la diffusione di Bibbie protestanti, si rimarcava altrettanto come questi sforzi si erano sem-

20. Stolberg, Friedrich Leopold, "Due lettere, una al conte di Smettau, l'altra a suo figlio", Memorie di Religione, di Morale e di Letteratura, vol. VI (1824), p. 89.

21. DE JouX, Pierre, "Lettere sull'Italia considerata riguardo alla religione. Articolo I", Memorie di Religione, di Morale e di Letteratura, vol. X (1826), p. 251.

22. Sulla volontà papale di tradurre immediatamente l'opera in italiano e per un elenco delle recensioni al lavoro del Cobbet, si veda FOA, Anna, Gli intransigenti..., p. 68. 
pre conclusi con palesi insuccessi, dovuti alla sostanziale fedeltà degli italiani alle autorità della propria tradizionale Chiesa, come si ricordava con esplicito compiacimento sulla Enciclopedia Ecclesiastica nel 1821:

"Sono già pochi anni che la società di Londra per mezzo di uno dei suoi emissari propagandisti ne fece giungere una enorme cassa a Palermo, e fu impegnata l'autorevole influenza della eccellentissima Signora Marchesa di Circello, [ma questa] negò di prestarsi al pio ministero, a meno che non avesse ricevuta l'approvazione dell'arcivescovo di quella metropoli, al quale in effetto ne presentò copia perché ne commettesse l'esame. Questa circostanza fece conoscere gli errori del libro, e i buoni effetti che dovevano attendersene. Ciò solo bastò perché la prelodata pia Signora tornasse ad assicurare gelosamente l'involto, ed il rimandasse in Malta"23.

In quegli anni, da parte legittimista venne a maturare la consapevolezza che, nonostante il rigore delle censure dei vari governi preunitari, la stampa era divenuta un formidabile strumento per la propaganda e la circolazione delle idee, e che l'efficacia di un giornale, di un opuscolo, di un libro risultava notevolmente accresciuta nella società del tempo. Convinti di rappresentare ancora la stragrande maggioranza dell'opinione pubblica italiana, i difensori del Trono e dell'Altare si impegnarono pertanto in una campagna editoriale di protezione e supporto a quelle istituzioni politiche e religiose, e a quelle norme sociali e comportamentali che avevano caratterizzato il vivere civile fino al travaglio rivoluzionario ${ }^{24}$. Peraltro, l'esempio veniva ancora dalla Francia, soprattutto dal giornale Le Conservateur fondato nel 1818 con la volontà di promuovere una radicale restaurazione del diritto della Chiesa ad ordinare la società, e quindi a condizionare il potere politico ${ }^{25}$.

Anche in Italia, all'inizio del secondo decennio del XIX secolo, si assistette pertanto ad una cospicua fioritura di periodici cattolici, a testimonianza di un sostanziale e diffuso risveglio cattolico a livello europeo, ben sintetizzato storiograficamente da Alfonso Omodeo quando rilevò che in quel periodo "il cattolicismo supera la fase della sua massima depressione che cade nella seconda metà del secolo XVIII, e ritrova le forze per operare nel mondo"26. Si tratta di un fenomeno geograficamente diffuso anche nel nostro paese, tale da infon-

23. Ventura, Gioacchino, "Sette religiose. Delle sette bibliche", Enciclopedia ecclesiastica e morale, vol. I (1821), p. 263.

24. Del Corno, Nicola, La formazione dell'opinione pubblica e la libertà di stampa nella pubblicistica reazionaria italiana del Risorgimento (1831-1847), Firenze, Le Monnier, 1997.

25. Omodeo, Adolfo, La cultura francese nell'età della Restaurazione, in Studi sull'età della Restaurazione, Torino, Einaudi, 1970, p. 147.

26. OMODEO, Adolfo, "Cattolicesimo e civiltà moderna nel secolo XIX", La Critica, vol. XXXIV (1936), p. 31. 
dere la speranza nei suoi sostenitori che potesse porre salde radici nel tessuto sociale italiano se si fosse riusciti a stabilire forme di collaborazione fra le varie realtà intransigenti locali, come dimostra una famosa lettera di Baraldi, direttore delle Memorie religiose a Ventura, direttore della Enciclopedia ecclesiastica del 4 agosto 1822, nella quale, presentando il proprio prodotto editoriale, invitava a scambiare reciprocamente le riviste in modo da poterle poi diffondere con maggiore capillarità:

"da molto tempo io nutrivo di entrare seco Lei in corrispondenza e procurarmi la Enciclopedia, che conosciamo solo per estratti, e ciò maggiormente dacché col principio dell'anno corrente s'intraprese da alcuni di noi la formazione e successiva pubblicazione di alcune Memorie di religione. Nel primo fascicolo delle medesime dando cenno d'altro giornale religioso che esce a Torino, accennai anche la sua Enciclopedia, ma ero impaziente di averla e di fare a Lei pure conoscere la nostra impresa allo stesso fine. [...] Mi prendo la libertà di accludere il manifesto, e quando si compiacerà indicarmi un recapito, mi farò pregio di inviarle i fascicoli. Così trattandosi d'opere guidate dallo stesso spirito, potremo aiutarci a vicenda, e se Ella favorisce di mandarmi anche per posta i numeri del suo Giornale, cui la prego sin d'ora di associarmi, li faremo viepiù conoscere dalle nostre parti con il mezzo delle Memorie. Se il Signore asseconda le nostre cause, i giornali ecclesiastici sono geograficamente ben disposti. Torino a settentrione, Napoli al meridione e Modena al centro"27.

Il riferimento correva appunto ai tre giornali sopraccitati che rappresentarono quanto di più incisivo la corrente intransigente seppe mettere in campo in quel frangente per divulgare idee e dottrine favorevoli all'alleanza fra Trono e Altare. A questi giornali, sia pure in tono minore per diffusione e qualità degli scritti si aggiunsero anche altre testate come, per limitarsi a citare le principali fra quelle di seconda fila, il Giornale ecclesiastico di Alessandria, la Pragmalogia Cattolica di Lucca, il Giornale degli apologisti della religione cattolica di Firenze, la Società dei Calobibliofili di Imola.

Legato strettamente in quel periodo ad Antonio Capece Minutolo, meglio noto come il Principe di Canosa -uomo di punta dello schieramento laico reazionario e per breve tempo ministro di polizia nel Regno del Due Sicile- il Ventura diede vita a Napoli nel giugno del 1821 alla Enciclopedia ecclesiastica e morale, prima rivista dell'ultramontanismo in Italia, che durò poco più di un anno riscontrando un vasto successo di diffusione soprattutto nello Stato borbonico, sebbene risulti difficile verificare la spontaneità dei suoi lettori, o se piuttosto fosse una lettura in qualche modo imposta dalle autorità politiche

27. FontanA, Sandro La controrivoluzione cattolica..., pp. 65-66. 
e religiose. Redatta nel consueto stile venturiano, ossia diretto, senza fronzoli, e soprattutto senza troppi riferimenti dotti che potessero appesantirne la lettura, l'Enciclopedia era nata con l'obiettivo di mettere in guardia la società dal contagio di nuove suggestioni rivoluzionarie, peraltro ben presenti nel territorio napoletano come i moti del luglio 1820 avevano appena dimostrato. Sul primo numero, nell'articolo in cui il Ventura spiegava la linea editoriale che avrebbe seguito la sua rivista, era evidente l'attacco a quelle società segrete, la Carboneria in testa, che svolgevano attività di proselitismo in funzione antiassolutista, e per questo venivano accusate dall'autore di voler dividere la società in partiti contrapposti, quando vi era semmai bisogno di una virtuosa unità politica e religiosa:

"Gli Stati non hanno bisogno, né di Lumi, né di Mura, né di Caldaje, né di Carboni. E qual vantaggio potrebbero attendersi esse mai da quelle associazioni tenebrose che, nate dal disordine, cresciute nel delitto, non tendono che a scindere in partiti le grandi famiglie dei popoli, e che, armando il cittadino contro il cittadino, preparano alla patria le desolanti sventure, figlie della divisione degli animi e dello scisma dei sentimenti? Gli Stati non hanno bisogno, principalmente al presente, che di virtù" ${ }^{\prime 28}$.

Nel suo giornale il giovane Ventura non diede spazio a particolari assunti dottrinali, o a sottili disquisizioni teorico-culturali, puntando invece sulla polemica diretta, esplicita e volta a denunciare l'incompiutezza della Restaurazione, soprattutto dal punto di vista dei valori. Per farla definitivamente finita con ogni retaggio rivoluzionario Ventura puntava, oltreché sull'autorità del binomio Trono e Altare, sulla fondamentale purezza di spirito del basso popolo, non contaminabile dalle pretese rivoluzionarie proprie della classe borghese. E proprio lo scontato apporto popolare alle sorti della cosiddetta "buona causa", ossia alla difesa dell'ordine tradizionale, forniva al Ventura la certezza della vittoria finale contro le forze della sovversione. Ma nell'ottobre del '22, con il ritorno al potere del ministro Luigi de' Medici, la cui linea politica era sostanzialmente ispirata alle nuove tendenze politiche moderate scaturite dal Congresso di Vienna, e il conseguente allontanamento del Canosa dal gabinetto ministeriale, la rivista venne soppressa.

Trasferitosi a Roma alla fine del '24, Ventura assunse la guida "informale" del Giornale ecclesiastico, il cui direttore ufficiale rimaneva il domenicano Francesco Ferdinando Jabalot, indirizzando la linea politica della testata su posizioni ultramontane e fieramente intransigenti, come scriveva trionfalmente al

28. Ventura, Gioacchino, "Programma", Enciclopedia ecclesiastica e morale, vol. I (1821), pp. III-IV. 
suo amico Canosa nel settembre del '25: "Da che il giornale è caduto nelle nostre mani, non vi è numero dove non si lodi Lamennais [...] per far crepare i giansenisti" ${ }^{29}$. Gli articoli del Ventura - improntati usando le sue stesse parole a dare una «nuova forma» al Giornale ${ }^{30}$ - suscitarono forti reazioni da parte delle autorità governative di diversi Stati, che chiesero con decisione alla Santa Sede un ritorno a quella moderazione nei toni e nei contenuti che aveva precedentemente contraddistinto la rivista. Il risultato di queste proteste si concretizzò nella chiusura del Giornale nel 1826.

Anche il direttore delle Memorie di Religione don Giuseppe Baraldi fu sicuramente una delle figure di primo piano del cattolicesimo intransigente italiano durante il primo trentennio del XIX secolo ${ }^{31}$. A partire dal 1820 egli cominciò a tenere ogni mercoledì presso la sua abitazione delle riunioni che avevano per oggetto discussioni religiose ed erudite, che lui stesso definiva "conferenze ecclesiastiche e letterarie"; coloro che vi prendevano parte erano studiosi di provata fede, laici e cattolici, in genere modenesi ${ }^{32}$. I principi fondamentali, che ispiravano tali riunioni, erano la lotta alle deviazioni antiromane, e di conseguenza la piena sottomissione all'autorità ecclesiastica in osservanza alle disposizioni sancite dal Concilio di Trento; Baraldi non volle però creare un'associazione religiosa, una vera e propria Amicizia Cattolica, secondo la formula piemontese, ma si accontentò di avere attorno a sé un gruppo di persone fidate, consapevole che il Duca di Modena Francesco IV non avrebbe tollerato la costituzione di una organizzazione che ai suoi occhi poteva tramutarsi in un partito, mentre avrebbe assecondato a livello meramente culturale una polemica religiosa e politica contro ogni intento innovatore.

Fu proprio durante una delle riunioni tenute a casa sua che Baraldi concepì l'idea di un giornale che avesse il compito di difendere Trono e Altare contro quelle tendenze moderne, reputate sovvertitrici degli equilibri sociali e politici. Così nel gennaio del 1822 iniziò la pubblicazione delle Memorie di Religione, di Morale e di Letteratura ${ }^{33}$, rivista culturale che usciva con cadenza variabile,

29. PIRRI, Pietro, "Il movimento lamennesiano in Italia. Nel centenario dell'Enciclica 'Mirari Vos': il P. Ventura e il Giornale Ecclesiastico", La Civiltà cattolica, vol. III (1932), p. 325.

30. Ventura, Gioacchino, "Introduzione al tomo terzo", Giornale ecclesiastico, vol. III (1825), p. 11.

31. Verucci, Guido, voce Giuseppe Baraldi, in Dizionario biografico degli italiani, Roma, Istituto della Enciclopedia italiana, 1963, vol. V, pp. 772-774.

32. FABRIANI, Severino, "Vita di Monsignor Baraldi", Continuazione delle Memorie di Religione, di Morale e di Letteratura, vol. III (1834), p. 108.

33. MAnni, Graziano, La polemica cattolica nel Ducato di Modena (1815-1861), Modena, STEM, 1968, pp. 95-183; Da CAMPagnola, Stanislao, Cattolici intransigenti a Modena 
ma prevalentemente bimestrale, destinata comunque a diventare in breve tempo uno dei periodici più importanti della controrivoluzione italiana nella prima metà dell' 800 , e che sopravvisse con alterne fortune fino al 1855, pertanto anche ben dopo la scomparsa del suo fondatore avvenuta nel 1832. Attorno a Baraldi si venne a formare una redazione che poteva contare su personaggi di primo piano dell'agguerrito mondo ultraconservatore modenese, molti di coloro che nel decennio successivo diedero vita, assieme al già citato principe di Canosa, alla ben più pugnace Voce della Verità. Fra i collaboratori occasionali più illustri delle Memorie vanno ricordati lo stesso Ventura, Antonio Rosmini, Antonio Bresciani e Félicité de Lamennais. Nel campo ultraconservatore, ciò che caratterizzò le Memorie rispetto ad altre riviste fu un tono assai meno battagliero, una maggiore attenzione verso l'erudizione soprattutto religiosa, una certa volontà del direttore, peraltro non condivisa da tutti i redattori, come ad esempio Marco Antonio Parenti, di tenersi lontano dalla insidiosa battaglia politica contingente, soprattutto per non mettere in difficoltà il proprio sovrano.

Fondato nel 1822 e diretto fino all'ultimo numero nel 1829 dal marchese Cesare d'Azeglio ${ }^{34}$, l'Amico d'Italia fu espressione tipica del mondo aristocratico piemontese, dipendente culturalmente dalla Francia. A Torino era già presente l'Amicizia cattolica, un'associazione che si proponeva di opporsi alla divulgazione della stampa avversaria diffondendo a mo' di propaganda libri e opuscoli controrivoluzionari scritti in modo semplice così da poter essere compresi da tutti ${ }^{35}$, e il giornale fu appunto emanazione della associazione nel concreto tentativo di collegare l'apologetica religiosa all'azione politica allo scopo di recuperare definitivamente la società al cattolicesimo più tradizionale. A differenza del gruppo modenese che aveva l'ambizione di rilanciare con forza la cultura cattolica come alternativa a quella illuminista, o del Ventura impegnato in una battaglia tutta politica contro il riproporsi di teorie rivoluzionarie, d'Azeglio ebbe l'obiettivo più modesto di divulgare la cultura tradizionalista francese nel nostro paese, accogliendo in ogni fascicolo numerose pagine degli autori transalpini. Laddove invece d'Azeglio e gli scrittori della rivista proposero i loro scritti originali, non si riscontrano discorsi particolar-

agli inizi della Restaurazione, Modena, Aedes Muratoriana, 1984, pp. 82-97; Del CoRno Nicola, Giuseppe Baraldi e le "Memorie di Religione" di Modena, in Lofficina letteraria e culturale dell'età mazziniana (1815-1870), a cura di Q. Marini, G. Sertoli, S. Verdino, L. Cavaglieri, Novi Ligure, Città del silenzio edizioni, 2013, pp. 183-193.

34. Pirri, Pietro, "Cesare d'Azeglio e gli albori della stampa cattolica in Italia", La Civiltà cattolica, vol. III (1930), pp. 193-212.

35. Bona, Candido, Le "Amicizie". Società segrete e rinascita religiosa (1770-1830), Torino, Deputazione subalpina di storia patria, 1962. 
mente incisivi; si trattò infatti di una stereotipa, quanto radicale polemica anti-liberale e anti-democratica che poco aggiungeva al discorso reazionario dei tempi, rappresentato con bel altra verve e incidenza già dal Canosa e dal Ventura. Da un punto di vista culturale e politico, il giornale torinese non raggiunse l'intensità e lo spessore delle altre due riviste sopraccitate; risultò una voce sì utile alla "buona causa" ma minore, senza quell'energia che invece i tempi richiedevano, come ben dimostra il severo giudizio che emerge in ambienti modenesi, quando, ad esempio, il Baraldi in più occasioni esternò in lettere private al Parenti nei primi mesi del 1822 la sua delusione per il giornale torinese: "è comparso il primo fascicolo dell'Amico d'Italia [...] non ne sono rimasto per niente soddisfatto. Edizione meschina, carta pessima, lingua barbara, articoli freddi, parole vuote", e ancora: "possibile che un amico non faccia conoscere a quel buon Marchese, animato da sì retto zelo, che quell'affettazione di stile semigotico [...] nuoce sommamente alla sostanza medesima del soggetto"36.

Come ha ben messo in luce un suo studioso, Angiolo Gambaro, la rivista fu troppo legata, in forma quasi mistica, alla tradizionale formula del Trono e dell'Altare per comprendere che i tempi si stavano evolvendo, e che pertanto le risposte da parte cattolico-intransigente dovevano necessariamente fare i conti con il progredire della storia ${ }^{37}$. Nel 1929, anziano -morirà l'anno successivostanco, deluso dai ripetuti urti con il governo piemontese che mal tollerava il suo essere lamennesiano, e peraltro ormai in rotta con lo stesso Lamennais dopo la pubblicazione del Des progrés de la Révolution et de la guerre contre l'Eglise, d'Azeglio decise di sospendere le pubblicazioni del giornale; nel prendere congedo dai lettori, nell'ultimo articolo scritto, l'autore ribadiva ancora una volta la sua fedeltà al passato, e di conseguenza come occorresse prendere le distanze dalle novità in politica come in religione: "non già che [si] debba far nuova teologia, perché novità e verità non s'incontrano insieme in teologia. Bensì le armi di questa debbono rivolgersi contro il nemico presente" 38 .

Anche nella produzione a stampa del più volte ricordato Principe di Canosa non è raro imbattersi in frequenti riferimenti al fondamento dell'imprescindibile ruolo della religione, o meglio della Chiesa cattolica, per una completa ristrutturazione dell'ordine legittimo e gerarchico nella collettività. Ricreata una salda convergenza tra religione e politica al fine di superare la crisi rivoluziona-

36. MAnni, Graziano, La polemica cattolica..., p. 176. Peraltro ci è noto come il d'Azeglio ricambiasse questi giudizi ben poco lusinghieri definendo il Baraldi e la redazione delle Memorie: "il Bibliotecario modenese con la sua sediziosa cricca"; PIRRI, Pietro, "Cesare d'Azeglio e gli albori"..., p. 202.

37. Gambaro, Angiolo, Sulle orme di Lamennais..., p. 105.

38. D’Azeglio, Cesare, "Il commiato del compilatore", L'Amico d'Italia, vol. XVI (1929), p. 382. 
ria, alla Chiesa veniva infatti conferita dal Canosa una posizione prioritaria rispetto a qualsiasi altra istituzione umana. Tale concezione teocratica della società, mutuata dal de Maistre del Du Pape ${ }^{39}$, induceva il Canosa a considerare il pontefice romano, oltre che investito delle sue naturali funzioni di guida spirituale della cristianità, anche di giudice assoluto delle questioni politiche temporali che potevano sorgere fra gli Stati, e pertanto stabile ed eterna garanzia di un tradizionale ordine politico e sociale: "ora però che siamo minacciati tanto dappresso dall'anarchia, e dalla dissoluzione civile, per carità chiamiamo il papa e facciamoci nelle dubbiezze nostre dirigere" 40 .

Questa visione finiva per indurre a considerare la fede principalmente come una necessità, dal momento che la personale credenza spirituale veniva fortemente condizionata dall'opportunità politica e civile di trovare nella religione, nei suoi dogmi e nelle sue autorità un prezioso sostegno per i troni vacillanti. Si veniva così a legittimare quell'assioma politico, che vedeva nel binomio Trono-Altare un sostanziato accordo politico tendente a combattere il comune avversario rappresentato da quei valori di derivazione illuminista protagonisti delle rivoluzioni del XVIII secolo. La religione doveva rifondare le monarchie legittime, le quali per conservarsi dovevano affidarsi senza particolarismi di sorta a un'unica religione e a un'unica Chiesa, poiché sia il papato che le monarchie risultavano le sole istituzioni che riunissero in sé le premesse per un'ordinata società: ossia autorità, continuità e unità ${ }^{41}$.

Il Canosa riteneva fondamentale l'alleanza fra Trono e Altare, soprattutto in tali momenti, vedendo nella Chiesa la struttura di base su cui rifondare i principi sociali e morali ormai pervertiti dall'opera di varie forze disgregatrici. Egli si trovava così inevitabilmente ad attribuire di frequente un essenziale significato di finalità politica alla Chiesa e alla stessa fede, tanto che in questa dimensione pragmatica e terrena rischiava di esaurirsi ogni personale convincimento religioso. $\mathrm{E}$ in tale indifferenza verso la dimensione metafisica ed escatologica della fede, anche nelle sue considerazioni a sfondo morale, la religione poteva apparire sminuita a precipuo strumento della causa terrena per cui egli combatteva. A questo riguardo risulta particolarmente interessante un'osservazione del suo biografo Walter Maturi, secondo cui "la fede religiosa del Canosa, pur raggiunta per personale convincimento e non succhiata

39. PRinCiPe di CANOSA, I piffari di montagna. Ossia cenno estemporaneo di un cittadino imparziale sulla congiura del principe di Canosa e sopra i Carbonari. Epistola diretta all'estensore del foglio letterario di Londra, seconda edizione accresciuta, Dublino [ma l'indicazione è falsa[, 1821, p. 171.

40. Ibid., pp. 172-173.

41. "Non essendoci ancora altr'ancora di salute per l'uman genere che il cattolicesimo e la legittimità", ribadiva infatti il CANOSA in uno dei suoi ultimi opuscoli, All'amico lettore, s.i.e. p. 33. 
passivamente col latte, fu limitata fin dalle origini da una duplice contaminatio: una contaminatio politica e una contaminatio cavalleresca"42. In effetti proprio questa dimensione cavalleresca, sebbene risulti secondaria rispetto a quella politica, aveva sempre influenzato nel bene e nel male i suoi comportamenti; anche nei suoi scritti, il Canosa amava infatti atteggiarsi a paladino della cristianità secondo modelli medioevali; appariva spesso disposto ad incrociare le armi contro chiunque osasse offendere o mettere in discussione i fondamenti della fede cattolica, quanto risoluto a mettere i propri averi e la propria vita a repentaglio con un ardore e una dedizione alla sacra causa che richiamavano gesta d'altri tempi.

Degna di più approfondita considerazione risulta la contaminatio fra politica e religione, in cui quest'ultima appariva frequentemente non come un'esperienza vissuta in maniera personale, in cui il singolo potesse trovare una superiore risposta alle proprie esigenze, bensì come un sistema inteso secondo schemi utilitaristici e interessati, ossia rivolto a finalità estranee, quando non opposte alla sua intima essenza. Lo strumento per la realizzazione di questo progetto era la Chiesa, che grazie ai suoi dogmi e ai suoi dettami morali, servendosi come tramite delle sue istituzioni, costituiva un mezzo imprescindibile per mantenere il popolo nell'obbedienza e sottomissione rispetto al potere costituito. Tale atteggiamento può però dar adito a interpretazioni e valutazioni contraddittorie; per un verso infatti la religione rischiava di venir inconsapevolmente considerata da lui come mero instrumentum regni, tanto da risultare assoggettata quasi completamente al potere politico. D'altra parte il Canosa voleva apparire come un rigoroso propugnatore di un papismo strettamente curialista ${ }^{43}$; e ciò gli imponeva di sostenere una sostanziale autonomia e una forte centralizzazione della struttura ecclesiastica, al fine di non concedere spazio eccessivo ai particolarismi delle varie chiese nazionali e ai giurisdizionalismi dei vari Stati, cosa che sarebbe stata inevitabile nel caso di un rapporto di subordinazione della Chiesa ai singoli regni, con il conseguente e concreto rischio d'infrangere l'unità del cattolicesimo e l'autorità assoluta del papa. Tale preoccupazione lo induceva a ribadire, ancora negli anni trenta, che "il sacerdozio deve essere indipendente e non ancella della politica" ${ }^{44}$.

42. MATURI, Walter, Il principe di Canosa, Firenze, Le Monnier, 1944, p. 2.

43. Le posizioni curialiste del Canosa rispetto alla politica regalista adottata dal Medici si trovano ben esplicitate in MATURI, Walter, Il Concordato del 1818 tra la Santa Sede e le Due Sicilie, Firenze, Le Monnier, 1929, pp. 21-24.

44. PRINCIPE DI CANOSA, La gazzetta Voce della verità condannata a morte ignominiosa senza appello con sentenza proferita a Parigi nell'aprile 1835 da ser cotale Niccolò Tommaseo e compagni per strage commessa dell'Antologia e per attentati contro la liberalesca settaria sovrana canaglia, Filadeldia [ma l'indicazione è falsa] 1835, p. 63. 
Ma lungi dall'essere teologo, il Canosa si dimostrava prioritariamente politico, sia pure di chiari sentimenti cattolici; di conseguenza nell'esporre le sue idee, finiva spesso per coinvolgere e adattare l'ortodossia secondo le sue convinzioni. A prevalere erano quindi gli interessi contingenti anche nella speculazione morale, e il Canosa conduceva con eccessiva disinvoltura fino agli estremi termini una personale concezione utilitaristica della religione. Egli così giungeva a rivolgere un solenne appello a protestanti, eterodossi, agnostici e atei, purché legittimisti, esortandoli all'assoluta necessità di credere per salvaguardare la stabilità dei troni. Infatti al termine del suo scritto politico più noto, uscito proprio nel 1820, I Piffari di montagna, si rivolgeva al suo interlocutore, ma più in generale all'intera collettività, sollecitandola a considerare e a sostenere la fede soprattutto come un obbligo per la sicurezza dei troni contro ulteriori rivolgimenti nel dopo Restaurazione: "se pure né al Papa volete credere, come anche a Gesù Cristo per la salute eterna, credetegli e crediamogli per quella di questa mondo"; per il Canosa infatti non "c'è mezzo termine o papa, o rivoluzione" 45 .

Il credere diventava così essenzialmente un'imposizione autoritaria; la ragione individuale doveva accettare la fede secondo una calcolata valutazione della sua utilità pragmatica, o peggio ancora per necessità e subordinazione ai dettami dell'ordine costituito. Ne conseguiva uno stravolgimento della missione della Chiesa; per adempiere alla sua funzione e realizzare i suoi scopi pastorali, più che alla persuasione e alla libera adesione ad una comunità religiosa, essa finiva per affidarsi al concreto sostegno dell'autorità costituita. Un governo autoritario e repressivo poteva pertanto risultare un necessario rimedio alla miscredenza della collettività: "quando un individuo, una classe di persone, un popolo ha meno timore di Dio, conviene che gli venga tanto più incusso quello del carnefice" ${ }^{46}$. Così Dio veniva accostato al carnefice in un tenebroso rapporto: "i settari non hanno paura di Dio, almeno abbiano quella del boia"47.

Ma non solo nelle grandi linee della sistemazione teorica la religione confluiva nella politica, o a essa risultava collegata. Il Canosa confidava infatti in un sempre più diretto ed esplicito intervento della Chiesa nelle questioni pratiche dei governi. Così in una lettera al Duca di Frammarino esortava alla simonia per far nominare un vescovo politicamente interessato ${ }^{48}$; auspicava in altra

45. Principe di Canosa, I piffari di montagna..., p. 172.

46. PRINCIPE Di CANOSA, Sulla proporzione delle pene secondo la diversità dei tempi, Modena, Tip. Camerale, 1831, p. 43.

47. Ibid. p. 65.

48. MATURI, Walter, Il principe di Canosa..., p. 62. Sempre dal lavoro del Maturi (pp. 226-227) si apprende il progetto del Canosa di utilizzare il clero come aiutante della polizia, rivelando agli agenti i segreti appresi in confessione da persone sospette. 
occasione il sicuro metodo della scomunica per individuare e isolare dal popolo gli avversari ${ }^{49}$; e giungeva infine a reclamare che il Papa, per stroncare i moti del '31 nelle Legazioni, "dovrebbe porre gli occhi sopra un sovrano devoto alla nostra Santa religione e intimando contro i settari una crociata, metter questo alla testa dei cattolici, dandogli il comando politico e militare"50.

Accanto a questi puntuali adempimenti di prassi politica, la Chiesa doveva tornare con più decisione ad assumere anche funzioni sociali, soprattutto nel campo pedagogico. L'educazione veniva fondamentalmente intesa come uno strumento di controllo sulla collettività, come si evidenzia dal suo famoso motto "meno istruzione e più religione" 51 ; il Canosa faceva infatti affidamento su di un'energica azione del clero per frenare l'immoralità e il progressivo sfacelo dell'umanità nel suo complesso. Secondo la sua pessimistica antropologia, l'uomo, già vile e corrotto a causa della primigenia caduta, aveva viepiù subito un'involuzione imputabile al dilagare dei falsi miti legati ad una presunta razionalità individuale, dimostrandosi così incapace di determinare realisticamente la propria esistenza, oltreché di definire in maniera autonoma la propria formazione spirituale e culturale. Dato che la filosofia, con le sue pretese rigeneratrici, aveva fallito nell'obiettivo di sostituirsi al soprannaturale, occorreva riaffermare come la religione fosse l'unica risorsa concessa all'umanità per correggere la naturale tendenza a regredire in una barbarie primordiale. In tale svalutazione delle iniziative fondate sulle capacità razionali, risultavano fondamentali i compiti della Chiesa, sola istituzione in grado di cogliere quelle fondamentali verità, negate invece alla conoscenza umana. Ma anche le idee del Canosa a proposito di quell'opera d'integrazione, che la Chiesa tramite l'esclusività dell'educazione avrebbe dovuto compiere, per ovviare alla solitudine degradante degli individui, finivano spesso per smarrire in una certa misura l'intendimento filantropico e sociale per venire assorbite entro moventi strettamente d'ordine politico; e come spesso gli accadeva, tali moventi risultavano indirizzati a fare dell'organizzazione religiosa un'istituzione finalizzata a conseguire risultati di conservazione e di freno. Infatti, sebbene avesse affermato come l'insegnamento religioso dovesse educare il popolo secondo precetti morali e civili, il Canosa non mancava però di precisare in cosa si risolvesse lo scopo prioritario dell'istruzione impartita dal clero: "per prevenire nei paesi cattolici

49. PRINCIPE di CANOSA, Sulla corruzione del secolo circa la mutazione dei vocaboli e delle idee. Lettera ad un amico, Italia, 1833, nota 1, p. 6.

50. PRINCIPE DI CANOSA, "Lettera sopra un articolo del Costituzionale", La voce della verità, vol. I (1832). I primi due volumi della La voce della verità non presentano la numerazione delle pagine.

51. PRincipe di Canosa, I piffari di montagna..., p. 116-117. 
le rivoluzioni contro i sovrani legittimi, parmi che basterebbe o avrebbe almeno grandissima forza l'istruzione fatta al popolo dai propri pastori sull'incompatibilità della religione nostra santissima con la rivoluzione medesima"52.

Nella elaborazione del suo programma politico teso a porre il credo religioso in una posizione di assoluta preminenza, l'influenza che il primo Lamennais ebbe sul Canosa risulta appieno confermata dalle stesse testimonianze del principe napoletano. Per intendere l'ammirazione per l'abate bretone e una iniziale dedizione alle idee da questo propugnate basta far riferimento al biglietto di presentazione che il Canosa inviò ai suoi amici modenesi in occasione del primo viaggio che il Lamennais compì attraverso l'Italia. Nella missiva il Canosa definiva il bretone "novello Sansone" ${ }^{53}$, investendolo enfaticamente di un ruolo biblico ben preciso, peraltro in pieno accordo con la sua maniera di concepire la tradizione religiosa.

Il Lamennais venne per la prima volta in Italia nell'estate del 1824; come evidenzia il Fontana già le tappe del suo tour italiano -Torino, Alessandria, Genova, Roma, Napoli, Modena- bene testimoniano quali fossero le città "lamennesiane" nel nostro paese, "i centri nevralgici del movimento" dove più erano attecchite le sue idee ${ }^{54}$. Lamennais fu molto colpito dall'entusiasmo, al limite del fanatismo, che suscitò la sua venuta in Italia; Ventura, nel recensire la nota opera del bretone sui rapporti fra religione e politica, scorgeva infatti nel pensiero e nell'azione dell'abate bretone un disegno divino per ripristinare il cattolicesimo nella sua funzione primaria di ordinatrice del consorzio umano: "il signor abate de Lamennais sembra essere stato scelto dalla provvidenza per annunziare alla società minacciata di una terribile dissoluzione, le più austere verità religiose e politiche che sono frattanto le cose che ne possono arrestare la ruina" ${ }^{55}$. A Modena, Lamennais si fermò solo un'ora, giusto il tempo per cambiare i cavalli della diligenza; nonostante questa breve fermata suscitò nel microcosmo modenese una incondizionata ammirazione e una fortissima emozione, ben testimoniata da questa entusiastica espressione di Baraldi: "Che umiltà! Che sapere! Che dolcezza!"

In seguito, l'evoluzione delle concezioni politiche di Lamennais mise sotto pressione gli intransigenti italiani: l'abate bretone da collante e simbolo della

52. Principe di Canosa, Catechismo sulla rivoluzioni, Modena, Soliani, 1832, Prefazione.

53. Bertoni, Giulio, Amicizie italiane del Lamennais, in Spunti, scorci e commenti, Genéve, Leo S. Olschki, 1928, p. 162

54. Fontana, Sandro, La controrivoluzione cattolica..., p. 211.

55. VentuRA, Gioacchino, "Recensione a De la considerée dans ses rapports avec l'ordre politique e civil", Giornale ecclesiastico, vol. I (1825) p. 264

56. MANNI, Graziano, La polemica cattolica..., p. 132. 
reazione divenne un fattore di divisione, fino a che le sue posizioni sempre più arditamente spinte oltre l'ortodossia cattolica furono una delle cause che portarono alla frammentazione del movimento reazionario sorto agli inizi degli '20. Con la fondazione del giornale filo-liberale L'Avenir nel 1830, Lamennais aveva rigettato ormai il principio legittimistico e conservatore, provocando le reazioni non solo di coloro -regalisti, gallicani, gesuiti- che già da tempo l'avversavano, bensì anche dei suoi vecchi sodali italiani, il Principe di Canosa in testa, che presero progressivamente le distanze in forme e modi diversi ${ }^{57}$. Ad esempio, nei mesi precedenti alla condanna papale dell'Avenir con la Mirari vos dell'agosto 1832 sia Ventura che Baraldi cercarono di difendere presso il papa il polemista francese, se non certamente le idee politiche-religiose, almeno la persona, suggerendo di tentare un recupero del bretone all'interno della Chiesa, mentre il giudizio del'arcivescovo genovese Lambruschini era improntato ad una severa condanna ${ }^{58}$.

Il turbamento che provocarono le evoluzioni del pensiero lamennesiano in Italia cadde in un momento già di per sé difficile per l'intransigentismo nostrano; nel 1826 la stella del Ventura era già iniziata a declinare in seguito alla pubblicazione delle sue lezioni tenute presso la cattedra di Diritto Pubblico all'Università romana della Sapienza ${ }^{59}$, dove aveva auspicato una maggiore solidarietà d'intenti fra papato e ultramontani a scapito delle diverse chiese nazionali, allarmando per questo ancora una volta alcuni gabinetti ministeriali europei, Austria e Francia in testa ${ }^{60}$. Forti a questo punto dell'appoggio di Metternich, i suoi oppositori -soprattutto i gesuiti- si fecero sentire presso le autorità pontificie affinché ritirassero al Ventura tutti gli incarichi che ricopriva a Roma, chiudendogli pure il Giornale Ecclesiastico e costringendolo ad una sorta di esilio nella piccola cittadina di Grottaferrata. Mentre, qualche anno dopo, nel giugno del 1828, il governo sabaudo, non immune da retaggi di regalismo, non apprezzò certamente la calorosa accoglienza che il d'Azeglio e il suo Amico d'Italia avevano riservato al Lamennais in visita a Torino ${ }^{61}$.

La prima ondata del movimento legittimista italiano, quella iniziata come reazione ai moti napoletani e torinesi, volgeva ormai al termine, pronta però a ritornare immediatamente qualche anno dopo con nuove parole d'ordine marcatamente più laiche, nuovi avversari, ossia i fautori dell'unità italiana -ciò

57. SANCIPRIANO, Mario, Lamennais in Italia..., pp. 16-17.

58. Manni, Graziano, La polemica cattolica..., pp. 153-157.

59. Colapietra, Raffaele, "Linsegnamento del P. Ventura alla Sapienza", Regnum Dei, vol. XVII (1961), pp. 230-259.

60. Tesini, Mario, Gioacchino Ventura..., pp. 37-38.

61. Gambaro, Angiolo, Sulle orme di Lamennais..., pp. 127-129. 
comportava che la difesa del Trono divenisse ora la priorità rispetto all'autonomia dell'Altare- e in un rinnovato contesto internazionale, dove la Francia ridiveniva terra di rivoluzione, e la prima guerra carlista spagnola forniva altre suggestioni e speranze, così come ulteriori occasioni di aspra polemica con le diplomazie europee.

Convinti sostenitori dell'immobilità strutturale di quelle istituzioni preunitarie legittimate dal volere divino e passate tutto sommato indenni dal travaglio rivoluzionario e napoleonico, i reazionari non si preoccuparono, nel decennio qui trattato, di elaborare un progetto di Italia alternativo rispetto a quelli messi in campo degli avversari, così come non reputavano possibile dinamiche da guerra civile tali da porre, in un futuro prossimo, italiani contro italiani su un campo di battaglia. Il ripetersi di sterili tentativi insurrezionali veniva infatti derubricato al rinnovarsi di una propensione rivoluzionaria che rimaneva settaria, e quindi circoscritta ad un numero limitato di congiurati senza coinvolgere il grosso della popolazione, considerata fedele allo status quo. Solo dopo le guerre d'indipendenza e il compimento dell'Unità il movimento reazionario e ultracattolico italiano sarà costretto a rimeditare su alcuni temi della contemporaneità politica, elaborando nuove visioni di patria, di nazione e dei rapporti fra Chiesa e Stato, fra religione e società, sicuramente legate alla tradizione passata, ma rivolte ad interpretare, e a non rifiutare preconcettualmente, le novità in corso in Italia ${ }^{62}$.

62. Si vedano a questo proposito, fra i più recenti contributi storiografici, Del CoRNo Nicola Patrie e nazione negli antiunitari, in Rileggere l'Ottocento, a cura di M.L. Betri, Torino, Carrocci, pp. 129-143; e i saggi di PinTo Carmine, La nazione mancata. Patria, guerra e resistenza negli scritti dei veterani borbonici del 1860-61, e MAROTTA Saretta, Cattolici "soci fondatori"? Il dibattito sulla partecipazione alla vita dello Stato unitario (1860-1886) in Antirisorgimento. Appropriazioni, critiche, delegittimazioni, a cura di M.P. Casalena, Bologna, Pendragon, 2013, rispettivamente pp. 87-125 e pp. 127-155. 